\title{
High-resolution computed tomography features and CT-guided microcoil localization of subcentimeter pulmonary ground- glass opacities: radiological processing prior to video-assisted thoracoscopic surgery
}

\author{
Zi-Xuan Wang ${ }^{1}$, Lin Li ${ }^{1}$, Zhe Zhang ${ }^{2}$, Guo-Hua Wang ${ }^{3}$, De-Mao Kong ${ }^{1}$, Xu-Dong Wang ${ }^{1}$, Fa Wang ${ }^{1}$ \\ ${ }^{1}$ Department of Interventional Radiology, ${ }^{2}$ Department of Thoracic Surgery, ${ }^{3}$ Department of Radiology, Qingdao Municipal Hospital, Qingdao \\ 266000, China \\ Contributions: (I) Conception and design: ZX Wang; (II) Administrative support: GH Wang; (III) Provision of study materials or patients: Z Zhang; (IV) \\ Collection and assembly of data: L Li; (V) Data analysis and interpretation: ZX Wang; (VI) Manuscript writing: All authors; (VII) Final approval of \\ manuscript: All authors. \\ Correspondence to: Zi-Xuan Wang, MD. Department of Interventional Radiology, Qingdao Municipal Hospital, 1 Jiao-Zhou Rd., Qingdao 266000, \\ China. Email: prince_room@sina.com.
}

Background: With the rapid development of high-resolution computed tomography (HRCT), low-dose CT scanning and video-assisted thoracoscopic surgery (VATS), smaller pulmonary nodules can be detected. Subcentimeter ground-glass opacities (GGOs) are extremely difficult to diagnose and accurately locate during VATS and in surgically resected specimens.

Methods: From September 2013 to September 2017, 42 subcentimeter GGO lesions $(\leq 1 \mathrm{~cm})$ in 31 patients who underwent CT-guided microcoil insertion followed by VATS resection were included. All HRCT images were assessed by two experienced radiologists, and CT-guided microcoil localization procedures were performed by two experienced interventional radiologists.

Results: A total of 42 subcentimeter GGOs included 28 malignancies (66.7\%) and 14 benign lesions (33.3\%). The diameter of malignant GGOs $(8.52 \pm 1.46 \mathrm{~mm})$ was significantly larger than that of benign lesions $(7.04 \pm 1.52 \mathrm{~mm})(\mathrm{P}<0.05)$. Seven patients had more than one GGO nodule. There were no significant differences in the location, composition, shape, margins, presence of air bronchograms, presence of the pleural indentation sign and presence of the vascular convergence sign between benign and malignant GGOs $(\mathrm{P}>0.05)$. All the localization procedures were performed successfully. A small pneumothorax occurred in 9 patients (21.4\%), and minor hemorrhage in the lung parenchyma occurred in 8 patients (19.0\%). All GGOs were easily identified during VATS and were definitively diagnosed.

Conclusions: Common HRCT features cannot be used as criteria for the differential diagnosis of subcentimeter benign and malignant pulmonary GGOs. CT-guided microcoil marking of these lesions prior to VATS is a feasible, safe, and effective procedure for the localization of subcentimeter pulmonary GGOs.

Keywords: Ground-glass opacity (GGO); pulmonary nodules; high-resolution computed tomography (HRCT); localization; microcoil

Submitted Oct 16, 2017. Accepted for publication Apr 12, 2018.

doi: $10.21037 /$ jtd.2018.04.87

View this article at: http://dx.doi.org/10.21037/jtd.2018.04.87 


\section{Introduction}

As the use of high-resolution computed tomography (HRCT) becomes widespread in clinical practice, we have increasingly encountered small or faint lesions on CT images (1). Low-dose CT scanning greatly increases the detection of small nodules during screening $(2,3)$. Groundglass opacity (GGO), a finding on HRCT, is defined as hazy increased attenuation of the lung with preservation of bronchial and vascular margins (4). It is a nonspecific finding that may be associated with several disorders (e.g., inflammatory diseases, atypical adenomatous hyperplasia, focal fibrosis and carcinoma). A previous study reported that $51.7 \%$ of detected lung cancers found during baseline screening were GGOs (5). Therefore, accurate differentiation between benign and malignant lesions before surgery was crucial, particularly for surgical planning and prognostic assessment.

Recently, the Fleischner Society provided recommendations for the management of GGOs (6). According to the statement, wedge, segmental, or subsegmental resections should be considered instead of a lobectomy. Video-assisted thoracoscopic surgery (VATS) is constantly evolving and becoming increasingly popular for the diagnosis and treatment of small pulmonary nodules and GGOs $(7,8)$. However, it is very difficult to palpate and identify small lesions during VATS. Preoperative localization with CT-guided percutaneous lung puncture technology helps to guide resection via VATS by placing a medium, such as a hookwire, methylene blue dye or a microcoil, in the lung for intraoperative localization of lesions $(9,10)$. Although there are several mediums used for localization of pulmonary lesions, CT-guided microcoil marking may be the most appropriate method for impalpable GGOs for several reasons.

In this study, we retrospectively analyzed 31 patients and evaluated the diagnostic performance of HRCT and CTguided microcoil localization for subcentimeter pulmonary GGOs prior to VATS.

\section{Methods}

\section{Study subjects}

From September 2013 to September 2017, a consecutive group of patients who underwent CT-guided microcoil insertion followed by VATS resection were included. Of these patients, those who had subcentimeter GGO lesions $(\leq 1 \mathrm{~cm})$ that had been confirmed by HRCT scans, surgical pathology (paraffin block) and a clinical diagnosis were included in the retrospective analysis in this study. The other selection criteria included a distance from GGOs to the visceral pleura of $\geq 5 \mathrm{~mm}$ and complete medical records. A CT-guided biopsy was not required before VATS.

This retrospective study was approved by the institutional ethics review board at Qingdao Municipal Hospital (No. 2018-006), and written informed consent was not required because of the strict maintenance of patient anonymity and the observational nature of the study.

\section{CT imaging}

Chest CT scans were performed using a Brilliance 64 (Philips Healthcare, Best, The Netherlands) or a Somatom Sensation 16 (Siemens Healthcare, Erlangen, Germany). All patients were scanned in the supine position at full inspiration for as long as possible. The imaging parameters were as follows: $100-300 \mathrm{~mA}, 120 \mathrm{kVp}$, pitch $0.875-1.500$, collimation $1.0-2.5 \mathrm{~mm}$ and rotation time $0.5-1.0 \mathrm{~s}$. Reconstruction thicknesses and intervals were $1-1.5$ and $1-1.5 \mathrm{~mm}$.

\section{Image assessment}

CT images were assessed by observing the lesion number, size (the longest dimension), location (lobe), composition (pure GGO or mixed GGO), shape (irregular or round/ oval), margins (lobulation, spiculation/cusp angles/spinelike processes), air bronchograms and adjacent structures (pleural indentation sign, vascular convergence sign).

In this study, GGO were classified as pure GGO (pGGO) or mixed GGO (mGGO) according to whether an internal solid component existed or not $(11,12)$. Marginal characteristics included lobulation and speculation/cusp angles/spine-like processes. Cusp angles and spine-like processes were both defined as structures extending from the lesion (13). Findings in adjacent structures included the pleural indentation sign and the vascular convergence sign.

\section{CT-guided microcoil localization}

All patients underwent CT-guided percutaneous pneumocentesis for localization in the supine, prone or lateral position on the same day prior to VATS. The patient's posture must be convenient for subsequent CT examinations and puncture procedures. A CT scan was performed to confirm the position of nodules before the 

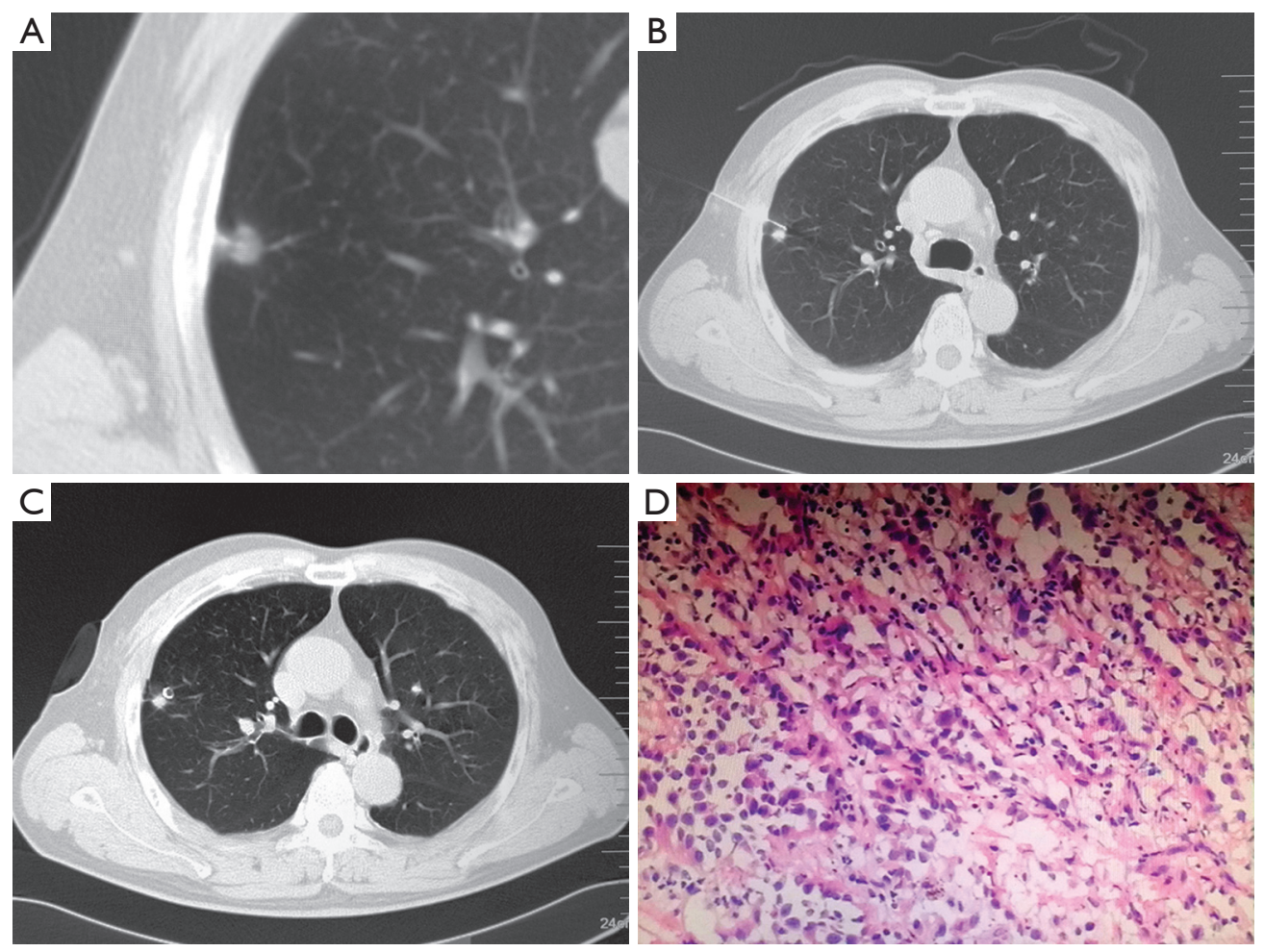

Figure 1 A 61-year-old male patient with a subcentimeter GGO underwent a CT-guided microcoil marking procedure and VATS. (A) Transverse lung window showing an 8.9-mm GGO with pleural indentation in the RUL; (B) the needle was inserted along the optimal path closest to the nodule; (C) a microcoil was placed next to the lesion; (D) surgical histopathological analysis indicated a MIA (H\&E stain, ×400). MIA, minimally invasive adenocarcinoma; GGO, ground-glass opacity; VATS, video-assisted thoracoscopic surgery; RUL, right upper lobe.

localization procedure (Figure $1 A$ ). After injection of $2 \%$ lidocaine local into the puncture site on the chest wall, a 20-gauge Chiba needle (Medical Device Technologies, Inc., Gainesville, FL, 32608, USA) was inserted under CT guidance. During the procedure, the needle pathway avoided the lesions, and the tip was inserted into the normal lung parenchyma close to the lesions (Figure 1B). Successful puncture was confirmed by a CT scan, and then a fiber-coated stainless steel microcoil that was $30-50 \mathrm{~mm}$ in length and 3-5 $\mathrm{mm}$ in diameter (William Cook Europe Aps, Bjaeverskov, Denmark) was pushed into the needle cannula by using the needle core. The needle core was advanced into the cannula up to the tip, and the microcoil was deployed into the lung parenchyma, where it assumed a tightly coiled ball configuration just beyond the tip of the needle. A localizing CT scan was performed to confirm the position of the microcoil, and the needle was then withdrawn (Figure 1C). The presence of pneumothorax or hemorrhage was assessed later. Complications were graded according to the National Cancer Institute Common
Terminology Criteria for Adverse Events Version 4.0 (CTCAE V4.0). In one patient with 6 GGOs, there were 3 GGOs in left upper lobe and 3 in the left lower lobe. All the GGOs were distributed in a small area. Therefore, we placed two microcoils in each lobe. Among the 4 coils, two were located in superficial positions for localization, and two were used to mark depths.

During the following VATS procedures, palpation for the microcoils was performed by bypassing the main operating port. For cases in which palpation was unsuccessful, C-arm $\mathrm{X}$-ray fluoroscopy was utilized to find the microcoil. When the marking microcoil was identified, a wedge resection was conducted if the lesions were adjacent to the pleural surface. Otherwise, a segmentectomy was performed. The incised specimen, including the lesion, the surrounding lung parenchyma and the marking microcoil, was sent for intraoperative frozen section histopathologic examination, and pathological examinations of paraffin slices of the specimen were performed after the resection. If the frozen section showed invasive adenocarcinoma or non-small 
Table 1 Demographic data of patients with benign and malignant GGOs ( $\mathrm{n}=31$ )

\begin{tabular}{lccc}
\hline Characteristics & Benign & Malignant & Significance \\
\cline { 3 - 4 } $\mathrm{N}(\%)$ & $9(29.0)$ & $22(71.0)$ & Statistics \\
Gender $(\mathrm{n})$ & & & $0^{\mathrm{a}}$ \\
Male & 6 & 14 & $0.233^{\mathrm{a}}$ \\
Female & 3 & $56.27 \pm 7.91$ & $\mathrm{t}=0.474$ \\
Age (year) & $57.78 \pm 8.32$ & 0.639 \\
\hline
\end{tabular}

${ }^{\mathrm{a}}, \chi^{2}$ test: Fisher's exact test. GGO, ground-glass opacity.

cell poorly differentiated adenocarcinoma, a segmental or subsegmental resection followed by lymph node sampling was performed, and the specimen was sent for pathological examination. If the lesion was benign, adenocarcinoma in situ, minimally invasive adenocarcinoma, or metastatic lung cancer, a chest tube was placed without any further resection. The patient was then recovered from anesthesia and taken to the recovery room.

\section{Data collection}

Demographic information (i.e., age and gender) and pathological results (i.e., surgical margins and pathological diagnosis) (Figure 1D) were collected from the medical records. CT image analysis was conducted independently by two experienced radiologists who were blinded to the patients' pathological results. Discrepancies in terms of the CT findings between observers were resolved by consensus. The CT-guided microcoil localization procedures and the assessment of results were performed by two experienced interventional radiologists. All data were recorded and entered into SPSS software for analysis.

\section{Statistical analysis}

The enumeration data are expressed as proportions, and the measurement data are expressed as the mean \pm standard deviation (SD). Statistical analysis of the difference between two means of continuous variables was performed using the independent samples $t$-test. The Pearson $\chi^{2}$ test, the continuity correction $\chi^{2}$ test and Fisher's exact test were used to compare categorical variables. A two-sided $\mathrm{P}$ value of 0.05 or less was considered a statistically significant difference. Statistical Package for the Social Sciences (SPSS)
(SPSS 15.0 for Windows, SPSS Inc., Chicago, IL, USA) was used to perform the statistical analysis of all data sets.

\section{Results}

In the study, 31 patients underwent HRCT scanning and CT-guided microcoil insertion (14 males and 17 females, mean age of $56.71 \pm 7.92$ years, range, $38-76$ years). Among all the 31 patients, a total of 42 subcentimeter GGOs were included in this study (28 malignancies $v s .14$ benign lesions). The demographic data are listed in Table 1. There were no demographic characteristics that were significantly associated with benign and malignant GGOs ( $\mathrm{P}>0.05)$.

HRCT findings of benign and malignant GGOs are reported in Table 2. The diameter of the lesions ranged from 4.1 to $10.4 \mathrm{~mm}$, as measured by HRCT. There was a statistically significant difference between benign GGOs and malignancies in terms of nodule size $(\mathrm{P}<0.05)$. Seven patients had more than one GGO nodule (six patients had two nodules, and one patient had six nodules). Eighteen patients had a single malignancy, and 6 patients had a single benign GGO. With respect to the other HRCT findings of the 42 subcentimeter GGOs, there were more mixed GGOs, round/oval GGOs, GGOs with a lobulation sign, and GGOs with air bronchograms in the malignant group, and there were more benign GGOs with spiculation/ cusp angles/spine-like processes. However, there were no significant differences in the location, composition, shape, margins, presence of air bronchograms, presence of the pleural indentation sign and presence of the vascular convergence sign between benign and malignant GGOs $(\mathrm{P}>0.05)$.

All the localization procedures were performed successfully (42 GGOs were marked with 40 microcoils, 
Table 2 HRCT findings of benign and malignant GGOs

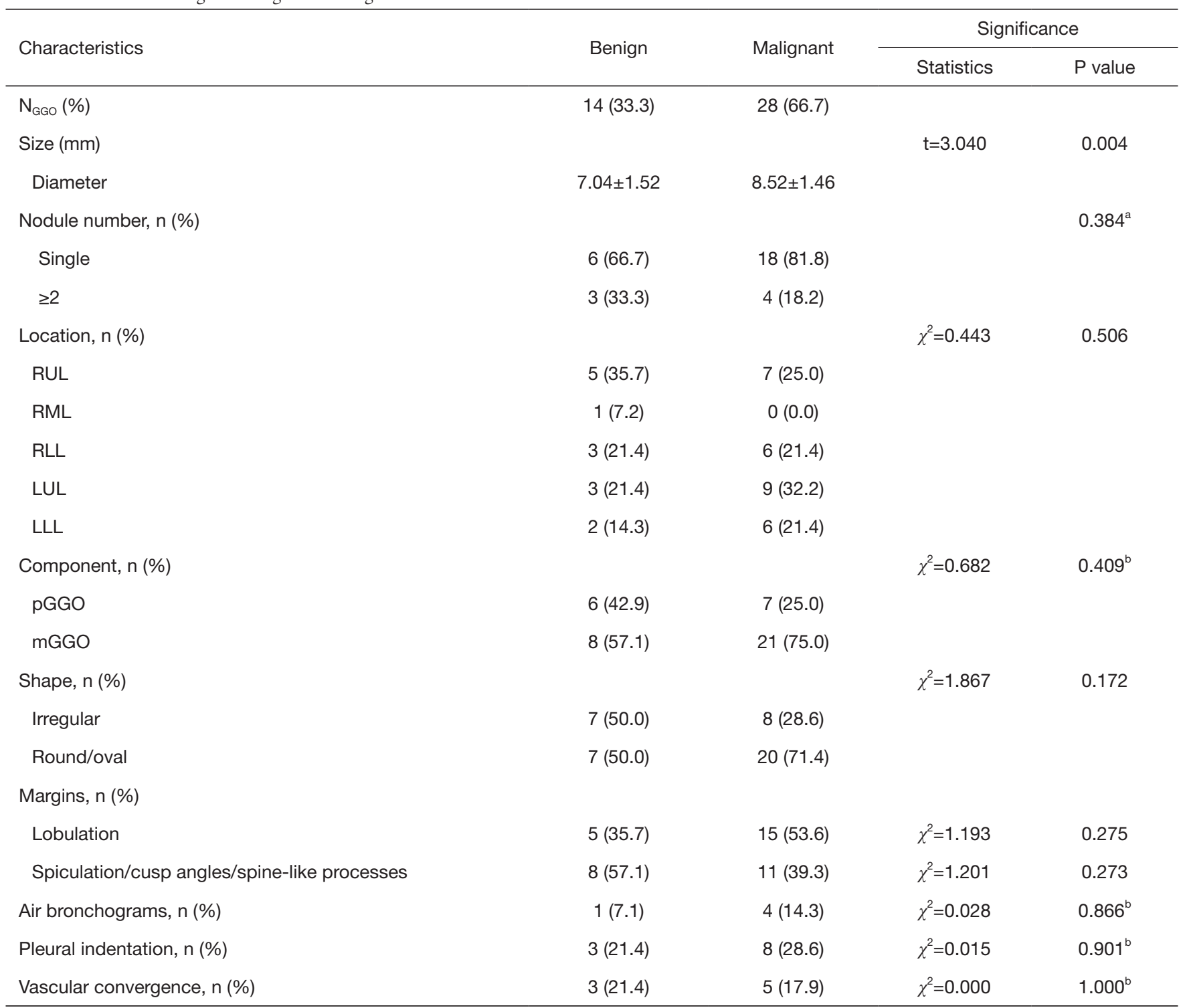

a, $\chi^{2}$ test: Fisher's exact test; ${ }^{b}, \chi^{2}$ test: continuity correction. LLL, left lower lobe; LUL, left upper lobe; RLL, right lower lobe; RML, right middle lobe; RUL, right upper lobe; HRCT, high-resolution computed tomography; GGO, ground-glass opacity.

for a success rate of $100 \%$ ) in this study. In terms of complications, a pneumothorax occurred in 9 patients (21.4\%), but no patients required drainage. Although hemorrhage in the lung parenchyma occurred in 8 patients $(19.0 \%)$, no patients were in serious condition, and no hemoptysis occurred. Patients only required observation. According to the CTCAE V4.0, all the complications were rated as grade 1 .

Forty-two lesions (100.0\%) were easily identified by locating the puncture hole and microcoil during VATS. The specimens were sent to the pathological department for examination. All GGOs were definitively diagnosed. The pathological results of the lesions are summarized in Table 3.

\section{Discussion}

Lung cancer is the leading cause of cancer-related deaths worldwide. Early detection and early intervention can 
Table 3 Pathological results of the lesions ( $n=42)$

\begin{tabular}{ll}
\hline Pathological result & $\mathrm{N}(\%)$ \\
\hline Surgical margin & $31(100.0)$ \\
Negative cases & $0(0.0)$ \\
Positive cases & \\
Pathological diagnosis & \\
Benign & $5(11.9)$ \\
AAH & $1(2.4)$ \\
DIPNECH & $3(7.1)$ \\
CIP & $2(4.8)$ \\
Fibrosis & $1(2.4)$ \\
Hamartoma & $2(4.8)$ \\
OP & \\
Malignant & $12(28.6)$ \\
AIS & $4(9.5)$ \\
IA & $5(11.9)$ \\
MIA & $5(11.9)$ \\
MLC & $2(4.8)$ \\
NSCPDA & \\
\hline AAH, atypical adenomatous hyperplasia; AIS, & \\
\hline &
\end{tabular}

$\mathrm{AAH}$, atypical adenomatous hyperplasia; AIS, adenocarcinoma in situ; DIPNECH, diffuse interstitial pulmonary neuroendocrine cell hyperplasia; CIP, chronic interstitial pneumonia; IA, invasive adenocarcinoma; MIA, minimally invasive adenocarcinoma; MLC, metastatic lung cancer; NSCPDA, Non-small cell poorly differentiated adenocarcinoma; OP, organized pneumonia.

improve the prognosis of lung cancer. With the rapid development of HRCT and low-dose CT scanning, smaller pulmonary nodules can be clearly detected (13) and more patients with small nodules that are highly suspicious of lung cancer can undergo resection with VATS (14). However, it is widely known that these lesions, especially subcentimeter GGO nodules $(\leq 1 \mathrm{~cm})$ that are small in volume and low in density, can be extremely difficult to diagnose before VATS and accurately locate during surgery and in surgically resected specimens, leading to problems for radiologists, surgeons and pathologists. Therefore, the differential HRCT features of benign and malignant subcentimeter GGOs and CT-guided localization procedures need to be investigated. In clinical practice, our thoracic surgeons have performed a variety of localization methods, such as injections of methylene blue dye and microcoil placement, and non-localization surgery for GGO patients. To evaluate
HRCT features and CT-guided microcoil localization methods, patients with subcentimeter GGOs were included in a study protocol at Qingdao Municipal Hospital, which is a general Hospital serving a population of 9.2 million in Qingdao (Shandong, China).

GGO lesions include benign and malignant lesions. Cho et al. (15) reported that among 330 GGOs resected without prior histologic confirmation, 314 GGOs (95.2\%) were malignancies, and a larger size was found to be an independent predictor of malignancy (OR, 1.086). Heo et al. (16) and Yamaguchi et al. (17) also proved that most GGO lesions were malignant (90\% and $87.2 \%$, respectively) and found that a larger size was correlated with malignancy in GGO nodules (16). In this study, 28 GGO nodules (66.7\%) from 22 patients were confirmed to be malignancies by surgical pathology. The diameter of malignant GGOs $(8.52 \pm 1.46 \mathrm{~mm})$ was significantly larger than that of benign lesions $(7.04 \pm 1.52 \mathrm{~mm})(\mathrm{P}<0.05)$. These results are consistent with the previously reported data

GGOs can be classified as pure GGOs (pGGOs) or mixed GGOs (mGGOs) according to the presence of solid components. Several previous studies (18-20) have found that mGGOs are more likely to be malignant. Matsuguma et al. (19) classified GGOs into 5 groups based on the proportion of the GGO area to the whole tumor shadow on HRCT scans and demonstrated that the proportion of GGO area on thin-section CT scans was a strong predictor of tumor aggressiveness. Most of the previous studies have focused on lung adenocarcinomas. In our study, we examined 42 GGOs that included benign and malignant lesions. There were more mGGOs in the malignant group, but no significant difference was found in the composition of benign and malignant GGOs ( $\mathrm{P}>0.05)$.

Fan et al. (13) retrospectively analyzed 82 pathologically and clinically confirmed focal GGOs and suggested that GGOs with lobulation, spiculation, spine-like processes, bronchus cut-off, other air-containing spaces, pleural indentation and vascular convergence were associated a greater than average likelihood of being malignant. Heo et al. (16) performed a retrospective analysis on 113 patients who underwent surgical resection without prior tissue diagnosis for highly suspicious pulmonary nodules and found that bubble lucencies and irregular margins were correlated with malignancy in GGO nodules $(\mathrm{P}<0.05)$. However, our results revealed that there were no significant differences in the shape, margins, presence of air bronchograms, presence of the pleural indentation sign and presence of the vascular convergence sign between benign 
and malignant GGOs in this sample. We believe that the differences in the baseline characteristics, i.e., GGO size and pathological type, may have resulted in the different outcomes. In addition, the HRCT features may not have been significant because the study is underpowered. The specific pathology may have resulted in a lack of power in the study.

During VATS, it is difficult to palpate and locate GGO lesions because these nodules are faint and have a GGO appearance or have a thin wall with a cavity. In addition, small lesions lying more than $2 \mathrm{~cm}$ deep in the lung parenchyma are difficult to palpate during surgery (21). Sometimes, more than one GGO is localized to one lung lobe, and although the lobe can be resected, these GGOs are generally too small to be found pathologically without localization prior to VATS (22). Therefore, it is necessary to perform a localization procedure before VATS. Currently, there are several localization methods available, which including hookwire localization (23), dye localization (24), microcoil localization (25), fiducial marker localization (26), contrast medium localization (27), radiotracer guided localization (28) and dual localization (29), etc. These methods use different localizing materials and have their own respective advantages. CT-guided microcoil localization is one of the most appropriate methods for subcentimeter GGOs for several reasons; it is associated with higher success rates [93-98.4\% (29)], less complications $[17.3 \%(25)]$ and less patient discomfort (30). In our study, 42 GGO nodules included 2 lung lobes with 2 GGOs and 2 lung lobes with 3 GGOs. All the subcentimeter GGOs were labeled successfully with stainless steel microcoils that were 0.035 inches in diameter. Only minor complications, i.e., a small pneumothorax and hemorrhage in the lung parenchyma, were detected on CT images. Several recent studies indicated that localization performed on the same day of surgery within a hybrid theatre is more safe and feasible (31-33).

There are several limitations to our study. First, a key limitation was that the study subjects were a convenience sample of patients. The population was relatively small both in the benign lesion group and in the malignancy group. Therefore, selection bias might have been inherent, and the results must be considered preliminary. Another limitation of this study was the potential selection bias from the retrospective design. Further prospective studies with large samples are necessary to verify the results of this study.

In conclusion, common HRCT features, e.g., component, shape, margins, air bronchograms, pleural indentation sign and vascular convergence sign, cannot be used as criteria for the differential diagnosis of benign and malignant subcentimeter pulmonary GGOs. CTguided microcoil marking of these lesions prior to VATS is a feasible, safe, and effective procedure for localization of pulmonary subcentimeter GGO.

\section{Acknowledgements}

None.

\section{Footnote}

Conflicts of Interest: The authors have no conflicts of interest to declare.

Ethical Statement: This retrospective study was approved by the institutional ethics review board at Qingdao Municipal Hospital (No. 2018-006), and written informed consent was not required because of the strict maintenance of patient anonymity and the observational nature of the study.

\section{References}

1. Swensen SJ, Jett JR, Hartman TE, et al. CT screening for lung cancer: five-year prospective experience. Radiology 2005;235:259-65.

2. Stang A, Schuler M, Kowall B, et al. Lung Cancer Screening Using Low Dose CT Scanning in Germany. Extrapolation of results from the National Lung Screening Trial. Dtsch Arztebl Int 2015;112:637-44.

3. Wiener RS, Gould MK, Arenberg DA, et al. An official American Thoracic Society/American College of Chest Physicians policy statement: implementation of lowdose computed tomography lung cancer screening programs in clinical practice. Am J Respir Crit Care Med 2015;192:881-91.

4. Patel VK, Naik SK, Naidich DP, et al. A practical algorithmic approach to the diagnosis and management of solitary pulmonary nodules: part 1 : radiologic characteristics and imaging modalities. Chest 2013;143:825-39.

5. Henschke CI, McCauley DI, Yankelevitz DF, et al. Early Lung Cancer Action Project: overall design and findings from baseline screening. Lancet 1999;354:99-105.

6. Naidich DP, Bankier AA, MacMahon H, et al. Recommendations for the management of subsolid pulmonary nodules detected at CT: a statement from the 
Fleischner Society. Radiology 2013;266:304-17.

7. Gonzalez D, de la Torre M, Paradela M, et al. Videoassisted thoracic surgery lobectomy: 3-year initial experience with 200 cases. Eur J Cardiothorac Surg 2011;40:e21-8.

8. Ng CS, Yeung EC, Wong RH, et al. Single-port sympathectomy for palmar hyperhidrosis with Vasoview Hemopro 2 endoscopic vein harvesting device. J Thorac Cardiovasc Surg 2012;144:1256-7.

9. Zaman M, Bilal H, Woo CY, et al. In patients undergoing video-assisted thoracoscopic surgery excision, what is the best way to locate a subcentimetre solitary pulmonary nodule in order to achieve successful excision? Interact Cardiovasc Thorac Surg 2012;15:266-72.

10. Shi Z, Chen C, Jiang S, et al. Uniportal video-assisted thoracic surgery resection of small ground-glass opacities (GGOs) localized with CT-guided placement of microcoils and palpation. J Thorac Dis 2016;8:1837-40.

11. Lee SM, Park CM, Goo JM, et al. Invasive pulmonary adenocarcinomas versus preinvasive lesions appearing as ground-glass nodules: differentiation by using CT features. Radiology 2013;268:265-73.

12. Liang J, Xu XQ, Xu H, et al. Using the CT features to differentiate invasive pulmonary adenocarcinoma from pre-invasive lesion appearing as pure or mixed groundglass nodules. Br J Radiol 2015;88:20140811.

13. Fan L, Liu SY, Li QC, et al. Multidetector CT features of pulmonary focal ground-glass opacity: differences between benign and malignant. Br J Radiol 2012;85:897-904.

14. Mogi A, Yajima T, Tomizawa K, et al. Video-Assisted Thoracoscopic Surgery after Preoperative CT-Guided Lipiodol Marking of Small or Impalpable Pulmonary Nodules. Ann Thorac Cardiovasc Surg 2015;21:435-9.

15. Cho J, Ko SJ, Kim SJ, et al. Surgical resection of nodular ground-glass opacities without percutaneous needle aspiration or biopsy. BMC Cancer 2014;14:838.

16. Heo EY, Lee KW, Jheon S, et al. Surgical resection of highly suspicious pulmonary nodules without a tissue diagnosis. Jpn J Clin Oncol 2011;41:1017-22.

17. Yamaguchi M, Furuya A, Edagawa M, et al. How should we manage small focal pure ground-glass opacity nodules on high-resolution computed tomography? A single institute experience. Surg Oncol 2015;24:258-63.

18. Aoki T, Nakata H, Watanabe H, et al. Evolution of peripheral lung adenocarcinomas: CT findings correlated with histology and tumor doubling time. AJR Am J Roentgenol 2000;174:763-8.

19. Matsuguma H, Yokoi K, Anraku M, et al. Proportion of ground-glass opacity on high-resolution computed tomography in clinical T1 N0 M0 adenocarcinoma of the lung: A predictor of lymph node metastasis. J Thorac Cardiovasc Surg 2002;124:278-84.

20. Li F, Sone S, Abe H, et al. Malignant versus benign nodules at CT screening for lung cancer: comparison of thin-section CT findings. Radiology 2004;233:793-8.

21. Congregado M, Merchan RJ, Gallardo G, et al. Videoassisted thoracic surgery (VATS) lobectomy: 13 years' experience. Surg Endosc 2008;22:1852-7.

22. Li M, Shen G, Gao F, et al. CT-guided fine-needle localization of ground-glass nodules in re-aerated lung specimens: localization of solitary small nodules or multiple nodules within the same lobe. Diagn Interv Radiol 2015;21:391-6.

23. Qi H, Wan C, Zhang L, et al. Early effective treatment of small pulmonary nodules with video-assisted thoracoscopic surgery combined with CT-guided dual-barbed hookwire localization. Oncotarget 2017;8:38793-801.

24. Muñoz-Largacha JA, Ebright MI, Litle VR, et al. Electromagnetic navigational bronchoscopy with dye marking for identification of small peripheral lung nodules during minimally invasive surgical resection. J Thorac Dis 2017;9:802-8.

25. Sui X, Zhao H, Yang F, et al. Computed tomography guided microcoil localization for pulmonary small nodules and ground-glass opacity prior to thoracoscopic resection. J Thorac Dis 2015;7:1580-7.

26. Sancheti MS, Lee R, Ahmed SU, et al. Percutaneous fiducial localization for thoracoscopic wedge resection of small pulmonary nodules. Ann Thorac Surg 2014;97:19148; discussion 1919.

27. Watanabe K, Nomori H, Ohtsuka T, et al. Usefulness and complications of computed tomography-guided lipiodol marking for fluoroscopy-assisted thoracoscopic resection of small pulmonary nodules: experience with 174 nodules. J Thorac Cardiovasc Surg 2006;132:320-4.

28. Ambrogi MC, Melfi F, Zirafa C, et al. Radio-guided thoracoscopic surgery (RGTS) of small pulmonary nodules. Surg Endosc 2012;26:914-9.

29. Kim KS, Beck KS, Lee KY, et al. CT localization for a patient with a ground-glass opacity pulmonary nodule expecting thoracoscopy: a mixture of lipiodol and India ink. J Thorac Dis 2017;9:E349-E53.

30. Lin MW, Chen JS. Image-guided techniques for localizing pulmonary nodules in thoracoscopic surgery. J Thorac Dis 2016;8:S749-S55.

31. Pang X, Xue L, Chen J, et al. A novel hybrid technique for 
localization of subcentimeter lung nodules. J Thorac Dis 2017;9:1107-12.

32. Yang SM, Ko WC, Lin MW, et al. Image-guided thoracoscopic surgery with dye localization in a hybrid operating room. J Thorac Dis 2016;8:S681-S9.

Cite this article as: Wang ZX, Li L, Zhang Z, Wang GH, Kong DM, Wang XD, Wang F. High-resolution computed tomography features and CT-guided microcoil localization of subcentimeter pulmonary ground-glass opacities: radiological processing prior to video-assisted thoracoscopic surgery. J Thorac Dis 2018;10(5):2676-2684. doi: 10.21037/ jtd.2018.04.87
33. Zhao ZR, Lau RW, Ng CS. Hybrid theatre and alternative localization techniques in conventional and singleport video-assisted thoracoscopic surgery. J Thorac Dis 2016;8:S319-27. 